\title{
A Novel Enhancer of the Wound Healing Process
}

\author{
The Fibroblast Growth Factor-Binding Protein
}

\author{
Sabine Werner \\ From the Department of Biology, Institute of Cell Biology, ETH \\ Zurich, Zurich, Switzerland
}

There are remarkable parallels between wound healing and cancer, at both the molecular and cellular levels, and it has been suggested that tumors use the wound healing response of the host to generate their own stroma. ${ }^{1} \mathrm{~A}$ particularly important component of the wound granulation tissue and the tumor stroma is the vasculature, which is essential for supplying the wound or tumor tissue with oxygen and nutrients. Thus, impaired angiogenesis is a hallmark of chronic, nonhealing ulcers, and stimulation of angiogenesis at the wound site is a promising treatment strategy. ${ }^{2}$ On the other hand, inhibition of angiogenesis is clinically used for the reduction of tumor growth. ${ }^{3}$ There are various growth factors and cytokines that stimulate angiogenesis in wounds and tumors. In particular, members of the vascular endothelial growth factor family are potent regulators of blood vessel and lymphangiogenesis. $^{2,3}$ In addition, certain members of the fibroblast growth factor (FGF) family are also involved in the control of angiogenesis, in particular in wounds and in tumors. ${ }^{4}$

FGFs comprise a family of 22 different members that regulate proliferation, migration, differentiation, and survival of various cell types. Therefore, they participate crucially in embryogenesis, tissue repair, and cancer. With the exception of FGF11-14, which act in the nucleus, the other FGFs exert their functions through binding to four different transmembrane receptor tyrosine kinases, designated FGF receptors 1-4 (FGFR1-4). ${ }^{5}$ The bioavailability of the secreted FGFs is rather limited, as most of them bind strongly to proteoglycans in the extracellular matrix. Therefore, they need to be released from the matrix before they can activate their receptors, and this is promoted by the fibroblast growth factor-binding proteins (FGF-BPs). Furthermore, FGFs are frequently expressed in small amounts, and enhancement of their receptor affinity, which is also achieved by FGF-BPs, may allow them to exert important biological functions at extremely low concentrations. In this issue of The American
Journal of Pathology, Tassi et $\mathrm{al}^{6}$ demonstrate that FGF$\mathrm{BP} 1$ promotes angiogenesis and wound repair through acceleration of FGF signaling.

\section{Fibroblast Growth Factor-Binding Proteins}

FGF-BPs comprise a family of three secreted proteins that act as FGF chaperones. The best-characterized member is FGF-BP1, which has been shown to bind to at least FGFs $1,2,7,10$, and $22 .^{7}$ On binding to the carboxyterminal part of FGF-BP1, FGFs are released from the extracellular matrix, and they are also protected from proteolytic degradation. In addition, when bound to FGFBPs, the affinity of FGFs for their transmembrane receptors is enhanced. ${ }^{7}$ Because FGFs are expressed in multiple types of cancer, ${ }^{8}$ the FGF chaperone function of FGF-BPs is likely to be of particular importance for cancer development and progression. Indeed, FGF-BP1 is highly expressed in the tumor cells of most carcinomas as well as in established carcinoma cell lines, and its expression correlates with a high microvessel density. ${ }^{7}$ The observed increased expression of FGF-BPs at early stages of the development of pancreatic and colorectal adenocarcinomas suggested that they could be used as a diagnostic screening method for detection of premalignant cancers, provided that they are released into the circulation of these patients. ${ }^{9}$ Most importantly, at least FGF-BP1 seems to play a functional role in tumorigenesis. Thus, it was shown in xenograft models for human cervical squamous cell carcinoma and colon adenocarcinoma that ribozyme-mediated suppression of FGF$\mathrm{BP}$ expression inhibits angiogenesis-dependent tumor growth. Therefore, FGF-BP1 appears to be required for

FGF research in the author's laboratory is supported by the Swiss National Science Foundation.

Accepted for publication September 6, 2011

CME Disclosure: The author did not disclose any relevant financial relationships.

Address reprint requests to Sabine Werner, Ph.D., Institute of Cell Biology, Swiss Federal Institute of Technology (ETH) Zurich, Schafmattstrasse 19, 8093 Zürich, Switzerland. E-mail: Sabine.werner@cell.biol.ethz.ch. 
efficient tumor growth in these cancer models, and it acts as an angiogenic switch. ${ }^{10}$

\section{FGFs and Wound Repair}

Given the important role of FGF-BP in tumor angiogenesis and growth, and the strong parallels between wound healing and cancer, it was tempting to speculate about a role of this protein in the repair process. Cutaneous wound healing is a highly regulated process that occurs in three partially overlapping phases: i) blood clotting and inflammation; ii) new tissue formation that involves reepithelialization and granulation tissue formation; and iii) tissue remodeling. Although this process is rather efficient in healthy individuals, it does not lead to complete regeneration in adult mammals. Rather, a scar remains with reduced tensile strength and elasticity and lack of all appendages. ${ }^{11}$ Several types of FGFs are expressed at the wound site, including FGF1, FGF2, FGF7, FGF10, and FGF22, and the expression of some of them is greatly increased at different phases of the repair process. ${ }^{12,13}$ This is functionally important because FGF2 knockout mice have impaired wound healing, ${ }^{14}$ and expression of a dominant-negative FGFR2 mutant in keratinocytes of transgenic mice resulted in a severe delay in wound re-epithelialization. ${ }^{15}$ Therefore, FGF signaling is obviously required for efficient healing. Thereby, different FGFs are likely to fulfill different functions: FGF2 seems to be particularly important for wound angiogenesis, whereas FGF7, FGF10, and FGF22, whose functions were blocked by the dominant-negative FGFR2 mutant, are important regulators of wound re-epithelialization. ${ }^{14,15}$ Because FGF-BP1 binds to multiple FGFs, it may well be involved in all these processes.

\section{Expression of FGF-BP1 in Healing Skin Wounds}

A role of FGF-BP1 in wound healing was first suggested by the rapid increase expression of FGF-BP1 expression after surgical wounding of human skin grafts. ${ }^{16}$ In another study, enhanced expression of FGF-BP1 was shown throughout the healing process of full-thickness excisional skin wounds in mice, and particularly strong expression of FGF-BP1 was observed in the hyperproliferative wound epidermis. ${ }^{17}$ In vitro studies with cultured keratinocytes suggested that various growth factors that are abundant at the wound site are responsible for the increase in FGF-BP expression in the wound epidermis. The predominant expression of FGF-BP1 by keratinocytes suggested that it accelerates the activity of FGFs that stimulate proliferation and migration of these cells, such as FGF7, FGF10, and FGF22. Indeed, these FGFs were identified as interaction partners of FGF-BP1, and the latter was shown to promote the activity of low concentrations of FGF7 and FGF10. ${ }^{17,18}$ Therefore, it seems likely that activation of FGF-BP1 expression in keratinocytes of healing wounds promotes re-epithelialization. In addition, FGF-BP1 may also act on cells of the granulation tissue (eg, endothelial cells), because it is a secreted protein and is also expressed in cells of the dermis and granulation tissue. To determine the functional importance of the enhanced FGF-BP1 expression in healing wounds, it will be essential to inhibit this protein at the wound site, for example, via neutralizing antibodies or small-interfering RNA-mediated knockdown. Because knockdown of FGF-BP1 in chicken embryos causes early embryonic lethality, ${ }^{19}$ it seems likely that knockout mice will also not be viable. However, wound healing studies in mice with a conditional knockout of FGF-BP in keratinocytes, fibroblasts, or endothelial cells would be an interesting approach to undertake for studying the role of endogenous FGF-BP1 in individual cell types for the healing process.

\section{FGF-BP1 Enhances Angiogenesis, Fibroblast Migration, and Wound Healing}

Although the role of endogenous FGF-BP1 in the wound healing process remains to be determined, Tassi et al ${ }^{6}$ now provide evidence for a possible therapeutic potential of this protein. This is of major medical importance because chronic nonhealing ulcers affect a large percentage of the population, in particular aged individuals. In addition, impaired healing that results in the formation of chronic ulcers is a frequent complication in diabetic patients or in patients treated with anti-inflammatory steroids or chemotherapy. This strongly reduces the quality of life of the affected individuals and may even require leg amputations. In addition, it creates a severe burden for the health care system. ${ }^{20}$ Thus, there is a strong need for the development of improved therapies for the treatment of impaired wound healing. Unfortunately, several approaches for local application of growth factors to chronic wounds have failed, most likely arising from the rapid degradation of the proteins at the wound site. ${ }^{21}$ Furthermore, a single growth factor usually affects a limited number of cell types and thus can only control certain aspects of the healing process. This is also the case for individual FGFs as described above. Therefore, acceleration of the activity of different FGF family members at the wound site appears as a promising strategy.

To determine whether FGF-BP1 has therapeutic potential for improvement of wound healing, Tassi et al ${ }^{6}$ generated transgenic mice expressing FGF-BP1 in an inducible manner (Tet-off system) under control of an ubiquitously active promoter. The inducible expression was necessary, as constitutive expression causes embryonic lethality. ${ }^{22}$ The consequences of FGF-BP1 upregulation for different processes involved in wound healing were tested, including fibroblast migration in vitro using scratch assays and angiogenesis in vivo using the Matrigel plug assay. Indeed, both processes were strongly stimulated in the presence of increased levels of FGF-BP1. Enhanced angiogenesis was also observed in healing skin wounds of FGF-BP1 transgenic mice, and the numbers of fibroblasts and macrophages at the wound site were also increased. These findings demonstrate that FGF-BP1 is a potent accelerator of wound granulation tissue formation. In addition, exogenously 
added FGF-BP1 enhances keratinocyte migration. ${ }^{16}$ Together with the finding that expression levels of the fgfbp1 transgene were particularly high in keratinocytes of the epidermis and the hair follicles, ${ }^{6}$ this finding indicates that re-epithelialization may also be accelerated in the FGF-BP1 transgenic mice. Indeed, the accelerated wound closure that was observed in these animals supports this hypothesis, although it remains to be determined whether this resulted from enhanced contraction and/or from enhanced re-epithelialization. A contribution of wound contraction seems likely because rodent wounds heal predominantly by contraction and because the number of contractile myofibroblasts was strongly increased on induction of FGF-BP1 expression. ${ }^{6}$

Interestingly, the effect of FGF-BP1 on wound repair was abolished when the mice were treated with an FGFR kinase inhibitor, strongly suggesting that the FGF-BP1induced acceleration of the wound healing process is FGF dependent. In the future, it will be interesting to identify the type of FGF(s) that is (are) positively regulated by FGF-BP1 in healing wounds. Wound healing studies in double-mutant mice expressing the fgf-bp 1 transgene and concomitantly lacking individual FGFs would answer this question. At least FGF1, FGF2, and FGF7 knockout mice could be used for this purpose, as they have no or only mild phenotypic abnormalities. ${ }^{5}$ Alternatively, individual FGFs could be inhibited at the wound site using neutralizing antibodies or small-interfering RNAs. The effect of FGF-BP1 on angiogenesis is particularly obvious; therefore, one would also like to know more about the quality of the new vessels. Does FGF-BP1 affect stabilization and functionality of the vessels? This could be tested by co-staining for endothelial cells and pericytes/smooth muscle cells and by in vivo perfusion assays (eg, with fluorescently labeled dextran), respectively. Finally, it should be determined whether the positive effect of FGF-BP1 on wound repair is accompanied by an increased scarring response, which might limit its therapeutic potential.

Independent of these open questions, the data presented by Tassi et al ${ }^{6}$ identify FGF-BP1 as a potent promoter of wound healing, even in healthy animals where the wound healing process is highly optimized. It will be exciting to determine the effect FGF-BP1 overexpression on wound healing in aged mice or in mice after induction of diabetes by streptozotocin treatment. Because diabetes is associated with impaired wound angiogenesis in mice and humans, ${ }^{2,20}$ the enhancement of FGF-BP1 levels may be particularly efficient under these conditions. Most importantly, the therapeutic potential of FGF-BP1 for impaired wound healing should be explored by application of recombinant protein or by selective production of FGF-BP1 at the wound site using a viral expression system. ${ }^{21}$ The carboxy terminus of FGF-BP1 is sufficient for FGF binding, thus, the use of smaller proteins could also be considered. The ultimate goal would be the use of FGF-BP1 for the treatment of chronic ulcers. Owing to the known instability of various growth factors in chronic wounds, ${ }^{21}$ which most likely concerns the FGFs as well, their stabilization by FGF-BP1 and the enhancement of the activity of low levels of growth factors is an exciting new perspective.

Finally, the therapeutic potential of FGF-BP1 may well go beyond the treatment of skin wounds. Thus, Tassi et $\mathrm{al}^{6}$ also demonstrated that FGF-BP1 enhances angiogenesis in the mouse ischemic hindlimb muscles. Furthermore, the expression of FGF-BP is increased in regenerating renal tubular epithelial cells, indicating a role in kidney repair. ${ }^{23} \mathrm{~A}$ strong increase in the expression of FGF-BP1 was also observed after spinal cord injury, and external FGF-BP1 stimulated FGF2-induced neurite outgrowth and enhanced neuronal survival in a PC12 neuronal culture model. ${ }^{24}$ These findings strongly suggest a role of FGF-BP1 in neuroprotection and repair. This hypothesis is further supported by the observation that FGF-BP down-regulation was associated with the failure to re-innervate the muscles during the progression of amyotrophic lateral sclerosis. ${ }^{18}$ Thus, FGF-BP1 may well emerge as a global player in tissue repair processes with an as yet underestimated therapeutic potential.

\section{References}

1. Schafer M, Werner S: Cancer as an overhealing wound: an old hypothesis revisited. Nat Rev Mol Cell Biol 2008, 9:628-638

2. Eming SA, Brachvogel B, Odorisio $T$, Koch M: Regulation of angiogenesis: wound healing as a model. Prog Histochem Cytochem 2007, 42:115-170

3. Ferrara N, Kerbel RS: Angiogenesis as a therapeutic target. Nature 2005, 438:967-974

4. Rusnati M, Presta M: Fibroblast growth factors/fibroblast growth factor receptors as targets for the development of anti-angiogenesis strategies. Curr Pharm Des 2007, 13:2025-2044

5. Ornitz DM, Itoh N: Fibroblast growth factors. Genome Biol 2001, 2:REVIEWS3005

6. Tassi E, McDonnell K, Gibby KA, Tilan JU, Kim SE, Kodack DP, Wilcox CS, William J. Welch, Gallicano GI, Johnson MD, Riegel AT, Wellstein A: Impact of fibroblast growth factor binding protein-1 (FGFBP1) expression on angiogenesis and wound healing. Am J Pathol 2011, 179:2220-2232

7. Abuharbeid S, Czubayko F, Aigner A: The fibroblast growth factorbinding protein FGF-BP. Int J Biochem Cell Biol 2006, 38:1463-1468

8. Turner N, Grose R: Fibroblast growth factor signalling: from development to cancer. Nat Rev Cancer 2010, 10:116-129

9. Tassi E, Wellstein A: The angiogenic switch molecule, secreted FGFbinding protein, an indicator of early stages of pancreatic and colorectal adenocarcinoma. Semin Oncol 2006, 33:S50-S56

10. Czubayko F, Liaudet-Coopman ED, Aigner A, Tuveson AT, Berchem GJ, Wellstein A: A secreted FGF-binding protein can serve as the angiogenic switch in human cancer. Nat Med 1997, 3:1137-1140

11. Gurtner GC, Werner S, Barrandon Y, Longaker MT: Wound repair and regeneration. Nature 2008, 453:314-321

12. Komi-Kuramochi A, Kawano M, Oda Y, Asada M, Suzuki M, Oki J, Imamura T: Expression of fibroblast growth factors and their receptors during full-thickness skin wound healing in young and aged mice. J Endocrinol 2005, 186:273-289

13. Werner S, Peters KG, Longaker MT, Fuller-Pace F, Banda MJ, Williams LT: Large induction of keratinocyte growth factor expression in the dermis during wound healing. Proc Natl Acad Sci U S A 1992, 89:6896-6900

14. Ortega S, Ittmann M, Tsang SH, Ehrlich M, Basilico C: Neuronal defects and delayed wound healing in mice lacking fibroblast growth factor 2. Proc Natl Acad Sci U S A 1998, 95:5672-5677

15. Werner S, Smola H, Liao X, Longaker MT, Krieg T, Hofschneider PH, Williams LT: The function of KGF in morphogenesis of epithelium and reepithelialization of wounds. Science 1994, 266:819-822

16. Kurtz A, Aigner A, Cabal-Manzano RH, Butler RE, Hood DR, Sessions RB, Czubayko F, Wellstein A: Differential regulation of a fibroblast 
growth factor-binding protein during skin carcinogenesis and wound healing. Neoplasia 2004, 6:595-602

17. Beer HD, Bittner M, Niklaus G, Munding C, Max N, Goppelt A, Werner $\mathrm{S}$ : The fibroblast growth factor binding protein is a novel interaction partner of FGF-7. FGF-10 and FGF-22 and regulates FGF activity: implications for epithelial repair. Oncogene 2005, 24:5269-5277

18. Williams AH, Valdez G, Moresi V, Qi X, McAnally J, Elliott JL, BasselDuby R, Sanes JR, Olson EN: MicroRNA-206 delays ALS progression and promotes regeneration of neuromuscular synapses in mice. Science 2009, 326:1549-1554

19. Gibby KA, McDonnell K, Schmidt MO, Wellstein A: A distinct role for secreted fibroblast growth factor-binding proteins in development. Proc Natl Acad Sci U S A 2009, 106:8585-8590

20. Sen CK, Gordillo GM, Roy S, Kirsner R, Lambert L, Hunt TK, Gottrup F, Gurtner GC, Longaker MT: Human skin wounds: a major and snowballing threat to public health and the economy. Wound Repair Regen 2009, 17:763-771
21. Fu X, Li X, Cheng B, Chen W, Sheng Z: Engineered growth factors and cutaneous wound healing: success and possible questions in the past 10 years. Wound Repair Regen 2005, 13:122130

22. McDonnell K, Bowden ET, Cabal-Manzano R, Hoxter B, Riegel AT, Wellstein A: Vascular leakage in chick embryos after expression of a secreted binding protein for fibroblast growth factors. Lab Invest 2005, 85:747-755

23. Ray PE, Tassi E, Liu XH, Wellstein A: Role of fibroblast growth factorbinding protein in the pathogenesis of HIV-associated hemolytic uremic syndrome. Am J Physiol Regul Integr Comp Physiol 2006, 290: R105-R113

24. Tassi E, Walter S, Aigner A, Cabal-Manzano RH, Ray R, Reier PJ, Wellstein A: Effects on neurite outgrowth and cell survival of a secreted fibroblast growth factor binding protein upregulated during spinal cord injury. Am J Physiol Regul Integr Comp Physiol 2007, 293:R775-R783 